\title{
Frequency of Blood Cultures Performed in a Community Hospital
}

\author{
Jaslyn M. Maurer ${ }^{1}$, Janice M. Burns², Mary R. Godfrey ${ }^{3}$, Carl M. Urban ${ }^{4,5}$, \\ Sorana Segal-Maurer ${ }^{4,6^{*}}$ \\ ${ }^{1}$ Franklin and Marshall College, New York Hospital Queens, Flushing, USA \\ ${ }^{2}$ Department of Nursing, New York Hospital Queens, Flushing, USA \\ ${ }^{3}$ Administration, New York Hospital Queens, Flushing, USA \\ ${ }^{4}$ Department of Medicine, New York Hospital Queens, Flushing, USA \\ ${ }^{5}$ New York University School of Medicine, New York, USA \\ ${ }^{6}$ Weill Cornell Medical College, Cornell University, New York, USA \\ Email: *sxsegalm@nyp.org
}

Received March 21, 2013; revised April 22, 2013; accepted April 30, 2013

Copyright (C 2013 Jaslyn M. Maurer et al. This is an open access article distributed under the Creative Commons Attribution License, which permits unrestricted use, distribution, and reproduction in any medium, provided the original work is properly cited.

\begin{abstract}
Background: Blood cultures (BCs) are obtained to identify etiologic organisms, demonstrate antibiotic efficacy, determine duration of treatment, and/or suggest further interventions. Published guidelines do not clearly state indications and timing for obtaining BCs. As a result, clinicians may obtain too many BCs, increasing cost and patient discomfort. Objective: To determine frequency of BCs performed at our hospital as part of a quality improvement project. Design: Retrospective review of all BCs submitted during a randomly selected month. Setting: A New York City 535-bed, university-affiliated community hospital. Measurements: Patient demographics and BC data were obtained from medical and laboratory records. Results: During the selected month, 2280 BCs were performed for 379 patients. Negative BCs were seen in 221 patients (58\%) with one-half having multiple BCs performed within 48 hours of admission and prior to obtaining results of initial BCs. Repeat BCs frequently did not reveal further pathogens among patients with either negative or positive initial BCs. Conclusions: Two-thirds of BCs were obtained from less than one-half of patients without added clinical utility. Often, BCs were repeated prior to results of initial BCs or repeated in patients receiving antibiotics in spite of known low yield following antibiotic initiation. Clinical assessment and review of initial BCs prior to obtaining further BCs is necessary. Staff education regarding appropriate clinical setting for BCs and indications for repeat BCs is required to maximize utilization of resources, improve diagnostic yield, and limit patient discomfort.
\end{abstract}

Keywords: Blood Cultures; Contamination; Repeat Blood Culture Timing

\section{Introduction}

Blood cultures (BCs) are obtained from patients prior to instituting antibiotic therapy in an effort to identify etiologic microorganisms, focus antibiotic therapy, determine optimal duration of treatment, and guide further interventions. Unfortunately, a small proportion of BCs yield true-positive results and inappropriately ordering BCs may be both wasteful and harmful [1-3]. In instances when BCs are contaminated by skin commensals, repeat BCs are obtained which may prolong hospitalization and unnecessary antibiotic administration [1,4,5]. Laboratory resource utilization review at New York Hospital Queens

"Corresponding author.
(NYHQ), a 535 bed university-affiliated community hospital, revealed a high number of BCs performed as compared to local and regional medical centers. Accompanying Clinical Microbiology Laboratory surveillance data revealed approximately $40 \%$ of hospitalized patients had an average of five sets of BCs (10 BC bottles) performed during their admission, representing two-thirds of all BCs obtained at NYHQ. Few of these were obtained in the setting of prior "true positive" BCs (i.e., gram-negative pathogens, streptococci, yeast, etc.) or initial contaminants (i.e., skin commensals). The current study was undertaken for a random chosen month in order to obtain more detailed data regarding timing of BCs and the incidence of repeat BCs as a baseline for a broader NYHQ 
Quality Improvement project aimed at optimizing laboratory resources, minimizing unnecessary patient testing, and defining potential points for intervention.

\section{Methods}

We retrospectively reviewed all BCs performed in a single randomly selected month using NYHQ Clinical Microbiology Laboratory daily log. Data collected included: patient name, location within the hospital at the time BCs were obtained, indication for BCs (obtained from laboratory order form), number of BCs performed, timing of BCs with respect to time of admission, number of positive and negative BCs, and type of bacteria isolated. A BC was defined as a single bottle (either aerobic or anaerobic) and a "set" as two BC bottles (one each of aerobic and anaerobic). Repeat BCs are defined as any BCs obtained after performance of the initial 4 BCs (2 sets).

All positive BCs at NYHQ are reviewed monthly routinely as part of Infection Control surveillance process and determinations of "true positive" or "contamination" are made using accepted definitions [4,6,7]. A determination of contamination is based on reported bacteria (including presence of skin flora including diphtheroids, coagulase-negative staphylococci, Bacillus sp., etc.), recovery of bacteria from few BCs (or different skin commensals from various simultaneous or sequential BCs), delayed detection of bacterial growth, no further growth isolated from subsequent cultures, and/or lack of physical and/or laboratory findings consistent with presence of infection. Further detailed chart review was not performed as part of this baseline data collection.

\section{Results}

During the randomly selected study period, there were 2280 BCs obtained from 379 patients, an average of 6 BCs (or 3 sets) per patient. The selected month was representative of Infection Control surveillance data for the 12 months prior with monthly positive BC rates of 4 to $6 \%$, contamination rate of approximately $1 \%$, and "true positive” BC rates of $3 \%-5 \%$.

Focusing on the number of single-patient BCs, 158 of 379 patients (42\%) had at least one positive BC. Of 158 patients with positive BCs upon admission, 14 patients had BCs with S. aureus, 22 patients with coagulasenegative staphylococci (CoNS) and the remainder with gram-negative bacteria (GNR), yeast, streptococci, or multiple pathogens isolated. Of 22 patients with CoNS, all were considered to be contaminants. The majority of repeat BCs were performed in the setting of initial positive BCs with S. aureus, CoNS, GNR, or streptococci isolates. However, none of these revealed further bacterial growth in the repeat BCs and outliers included patients with more than 20 BCs performed. Figure 1 represents the number of repeat blood cultures performed after the initial blood cultures has specific pathogens (such as S. aureus, CoNS, GNRs, other). For example, in patients with initial blood cultures with Staphyloccocus aureus, follow-up blood cultures were repeated more than 3 sets in 6 patients, $<2$ sets in 3 patients, 5 sets in 1 patient, and more than 6 sets in 3 patients. It's important to differentiate the number of blood cultures repeated depending on the initial pathogens. It is appropriate to repeat BC in the setting of Staphylococcus aureus and possibly CoNS but not GNRs.

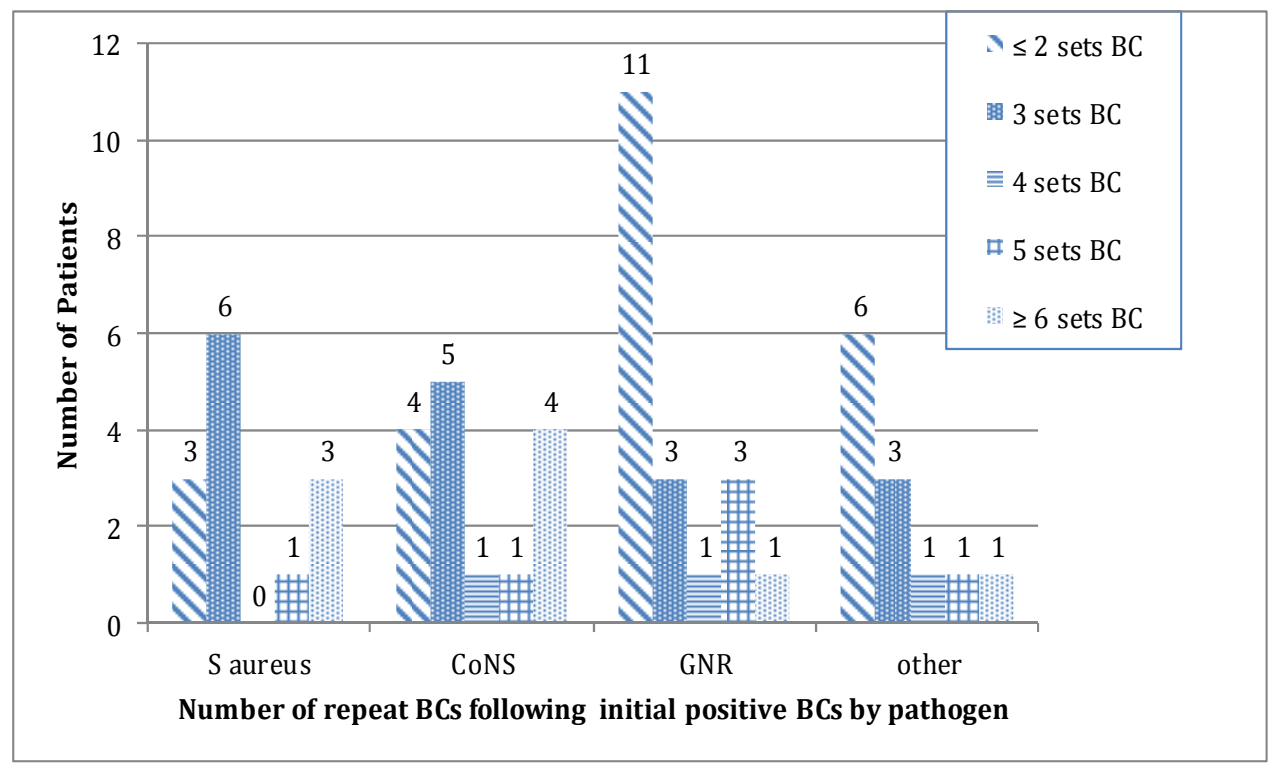

Figure 1. Repeat BCs among patients with initial positive BCs upon admission ( $=158$ of total 379 patients) ${ }^{*}$. BC: blood cultures, CoNS: coagulase-negative staphylococci, GNR: gram-negative rods. * All repeat BC revealed no further growth. 
Of 221 patients with initial negative BCs, four patients were not evaluable due to insufficient information. Of the remaining 217 patients, repeat BCs (4 bottles or 2 sets) were obtained from 128 patients (58\%) within 48 hours of admission and prior to any results available from the initial BCs obtained upon admission (Figure 2). More than two sets (4 bottles) were obtained from 56 patients (33 and 23 patients had three and four sets repeated within 48 hours, respectively) and 37 patients underwent multiple repeat BCs more than 72 hours following admission (on consecutive days). Of 217 patients, more than 6 BCs (3 sets) were performed daily in 70 patients in spite of no further growth in repeat BCs.

\section{Discussion}

Blood cultures are an important diagnostic modality for seriously ill patients. Over 200,000 cases of bacteremia occur yearly with an associated mortality of over $50 \%$ $[1,8]$. Indications for drawing BCs are broad and illdefined although opinions exist as to optimal timing of blood cultures [1,2,9]. Clinical parameters alone have not been helpful for the prediction of bacteremia and thus are not always useful guides for the identification of patients from whom blood samples should be obtained for culture $[1,10]$. It is not uncommon for a febrile patient with an infection to have persistent fever during the initial 72 hours in spite of receiving appropriate antibiotics. Obtaining repeat BCs in this setting has low yield of growth $[1,10]$.

Data exists regarding excessive BCs drawn, especially in teaching hospitals, with a disappointing overall yield [11]. Negative BCs do not always lead to a change in antibiotics and, thus, may prove clinically unnecessary [12]. When faced with immuno-competent patients (as well as those not considered to have endocarditis), it may be prudent to review the "pre-test probability" of bacteremia in each clinical circumstance in order to decide about the utility of performing BCs and not to rely on single physical findings [1-3]. In addition, yield of positive BCs from patients with diagnoses of skin and soft tissue or respiratory infections are typically low [13].

Although guidelines exist for drawing initial BCs (frequency, number, volume), none exist for repeating BCs, especially in the setting of prior antibiotic administration [14]. Among hospitalized, non-intensive care unit patients, antibiotic treatment within one week of BCs proved to be an independent predictor of negative BCs [13]. Even among neutropenic febrile patients, yield of positive BCs decreases from $7.4 \%$ to $1.7 \%$ following antibiotic administration [15]. Although the most common reason for repeating BCs is persistent fever, new pathogens are rarely identified [1,16-18]. Settings where repeating BCs may be indicated include: 1) clinical suspicion of a new septic episode; 2) suspected endocarditis; 3) need to demonstrate eradication of organisms (i.e., endocarditis and when bacteremia is due to particular pathogens such as $S$. aureus, Candida species, multidrug resistant GNR, etc.); 4) confirmation of response to treatment (i.e., endovascular infections); and 5) diagnosis of catheter-related infection (i.e., BCs from catheter and separate peripheral site) [8,9,16,19-21]. Settings where repeat BCs should be avoided include: 1) persistent fever without additional findings; 2) leukocytosis without clinical findings; and 3) focal infection without systemic signs or symptoms [16].

The purpose of this study was to determine the frequency of repeat BCs as part of a larger Quality Improvement project to evaluate optimization of laboratory resources and improved patient care. Chart review was

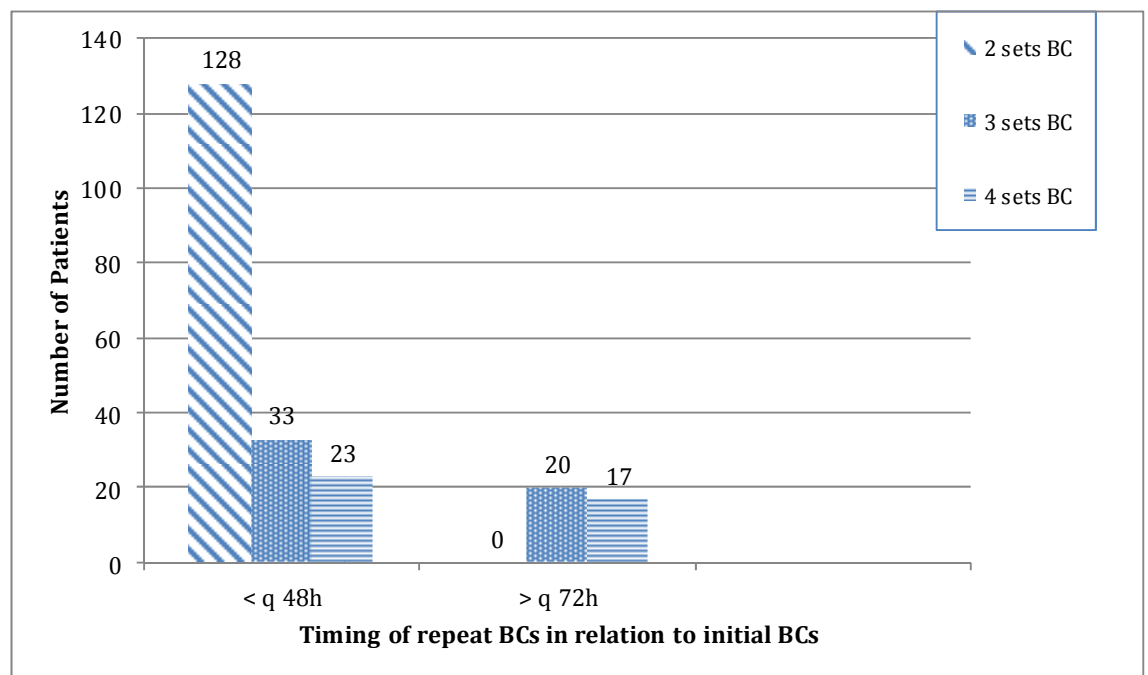

Figure 2. Repeat BCs among patients with initial negative BCs upon admission ( $n=221$ of 379 patients) ${ }^{*}$. BC: blood cultures; *All repeat BC remained without growth. 
not undertaken since the study was not intended to demonstrate effectiveness of BCs but rather to examine frequency of BCs in view of our discrepant numbers compared to other area facilities with similar acuity of hospitalized patients. Although our study was limited by lack of detailed chart review, nevertheless, it was remarkable for the finding that in the large proportion of cases, healthcare providers obtained BCs prior to waiting for results or performed multiple repeat BCs over the space of days when these were not indicated (and in spite of continued negative yield).

In a prior quality improvement pilot study undertaken by the Infectious Diseases residents at our institution, members of the medical housestaff were contacted in regard to positive BCs to ensure timely information communication as well as appropriate selection of antibiotics. In addition to improving patient care by adjusting antimicrobial therapy in over two-thirds of patients, unnecessary repeat BCs were eliminated for a large number of patients (Kopacz et al., unpublished results). Furthermore, multiple studies suggest use of dedicated phlebotomy teams which employ standardized BC techniques to limit contamination and resulting unnecessary interventions [4$7,22,23]$.

Our current study offers an opportunity for education of hospital staff regarding general guidelines for obtaining BCs as well as indications for repeat BCs in an effort to appropriately utilize limited resources and maximize patient safety. Future role of inflammatory markers (e.g., procalcitonin) in guiding BC performance and/or timing for repeat BCs needs to be further explored and may help limit unnecessary testing.

\section{REFERENCES}

[1] B. Coburn, A. M. Morris, G. Tomlinson and A. S. Detsky, "Does This Adult with Suspected Bacte Remia Require Blood Cultures?” JAMA, Vol. 308, No. 5, 2012, pp. 502511. doi:10.1001/jama.2012.8262

[2] N. I. Shapiro, R. E. Wolfe, S. B. Wright, R. Moore and D. W. Bates, "Who Needs a Blood Culture? A Prospectively Derived and Validated Prediction Rule,” Journal of Emergency Medicine, Vol. 35, No. 3, 2008, pp. 255-264. doi:10.1016/j.jemermed.2008.04.001

[3] C. C. Lee, C. J. Wu, C. H. Chi, N. Y. Lee, P. L. Chen, H. C. Lee, C. M. Chang, N. Y. Ko and W. C. Ko, "Prediction of Community-Onset Bacte Remia among Febrile Adults Visiting an Emergency Department: Rigor Matters," Diagnostic Microbiology and Infectious Diseases, Vol. 73, No. 2, 2012, pp. 168-173. doi:10.1016/j.diagmicrobio.2012.02.009

[4] M. P. Weinstein, "Blood Culture Contamination: Persisting Problems and Partial Progress," Journal of Clinical Microbiology, Vol. 41, No. 6, 2003, pp. 2275-2278. doi:10.1128/JCM.41.6.2275-2278.2003

[5] R. B. Schifman, C. L. Strand, F. A. Meier and P. J. Ho- wanitz, "Blood Culture Contamination: A College of American Pathologists Q-Probes Study Involving 640 Institutions and 497134 Specimens from Adult Patients," Archives of Pathology and Laboratory Medicine, Vol. 122, No. 3, 1998, pp. 216-221.

[6] M. L. Towns, W. R. Jarvis and P. R. Hsueh, "Guidelines on Blood Cultures,” Journal of Microbiology Immunology and Infection, Vol. 43, No. 4, 2010, pp. 347-349. doi:10.1016/S1684-1182(10)60054-0

[7] K. K. Hall and J. A. Lyman, "Updated Review of Blood Culture Contamination," Clinical Microbiology Reviews, Vol. 19, No. 4, 2006, pp. 788-802. doi:10.1128/CMR.00062-05

[8] C. J. Grace, J. Lieberman, K. Pierce and B. Littenberg, "Usefulness of Blood Culture for Hospitalized Patients Who Are Receiving Antibiotic Therapy," Clinical Infectious Diseases, Vol. 32, No. 11, 2001, pp. 1651-1655. doi:10.1086/320527

[9] C. W. Stratton, "The Use and Abuse of Blood Cultures," Infectious Disease Newsletter, Vol. 10, No. 4, 1991, pp. 28-31.

[10] Y. F. Van der Heijden, G. Miller, P. W. Wright, B. E. Shepherd, T. L. Daniels and T. R. Talbot, "Clinical Impact of Blood Cultures Contaminated With CoagulaseNegative Staphylococci at an Academic Center,” Infection Control Hospital Epidemiology, Vol. 32, No. 6, 2011, pp. 623-625. doi:10.1086/660096

[11] J. M. Darby, P. Linden, W. Pasculle and M. Saul, "Utilization and Diagnostic Yield of Blood Cultures in a Surgical Intensive Care Unit,” Critical Care Medicine, Vol. 25, No. 6, 1997, pp. 989-994. doi:10.1097/00003246-199706000-00016

[12] B. P. Ehrenstein, T. Jarry, H. J. Linde, J. Scholmerich and T. Gluck, "Low Rate of Clinical Consequences Derived from Results of Blood Cultures Obtained in an Internal Medicine Emergency Department," Infection, Vol. 33, No. 5-6, 2005, pp. 314-319. doi:10.1007/s15010-005-5065-5

[13] B. P. Ehrenstein, V. Ehrenstein, C. Henke, H. J. Linde, B. Salzberger, J. Schölmerich and T. Glück, "Risk Factors for Negative Blood Cultures in Adult Medical Inpatients-A Retro Spective Analysis,” BMC Infectious Diseases, Vol. 8, 2008, pp. 148-152. doi:10.1186/1471-2334-8-148

[14] M. Aronson and D. Bor, "Blood Cultures," Annals of Internal Medicine, Vol. 106, No. 2, 1987, pp. 246-253. doi:10.7326/0003-4819-106-2-246

[15] N. Bartlett and M. Naughton, "Assessing the Utility of Repeat Blood Cultures Obtained during Neutropenic Fever," American Society of Clinical Oncology Annual Meeting, 1999, Abstract No. 2269.

[16] M. S. Tabriz, K. Riederer, J. Baran and R. Khatib, "Repeating Blood Cultures during Hospital Stay: Practice Pattern at a Teaching Hospital and a Proposal for Guidelines," Clinical Microbiology and Infection, Vol. 10, No. 7, 2004, pp. 624-627. doi:10.1111/j.1469-0691.2004.00893.x

[17] F. J. Roberts, “The Value of the Second Blood Culture," Journal of Infectious Diseases, Vol. 168, No. 3, 1993, pp. 795-796. doi:10.1093/infdis/168.3.795 
[18] S. Shafazand and A. B. Weinacker, "Blood Cultures in the Critical Care Unit: Improving Utilization and Yield," Chest, Vol. 122, No. 5, 2002, pp. 1727-1736. doi:10.1378/chest.122.5.1727

[19] P. H. Chandrasekar and W. J. Brown, "Clinical Issues of Blood Cultures,” Archives of Internal Medicine, Vol. 154, No. 8, 1994, pp. 841-849.

doi:10.1001/archinte.1994.00420080023003

[20] J. M. Mylotte and A. Tayara, "Blood Cultures: Clinical Aspects and Controversies," European Journal of Clinical Microbiology and Infectious Disease, Vol. 19, No. 3, 2000, pp. 157-163. doi:10.1007/s100960050453

[21] F. Y. Chang, B. B. MacDonald, J. E. Peacock Jr., D. M. Musher, P. Triplett, J. M. Mylotte, A. O’Donnell, M. M. Wagener and V. Yu, "A Prospective Multicenter Study of
Staphylococcus Aureus Bacteremia: Incidence of Endocarditis, Risk Factors for Mortality, and Clinical Impact of Methicillin Resistance,” Medicine, Vol. 82, No. 5, 2003, pp. 322-332.

doi:10.1097/01.md.0000091185.93122.40

[22] F. L. Weinbaum, S. Lavie, M. Danek, D. Sixsmith, G. F. Heinrich and S. S. Mills, "Doing It Right the First Time: Quality Improvement and the Contaminant Blood Culture,” Journal of Clinical Microbiology, Vol. 35, No. 3, 1997, pp. 563-565.

[23] L. G. Bekeris, J. A. Tworek, M. K. Walsh and P. N. Valenstein, "Trends in Blood Culture Cotamination: A College of American Pathologists Q-Tracks Study of 356 Institutions," Archives of Pathology and Laboratory Medicine, Vol. 129, No. 10, 2005, pp. 1222-1225. 